\title{
Influence of physical training after a myocardial infarction on left ventricular contraction mechanics
}

\author{
Marcio Lima ${ }^{1}$, Talia Falcão Dalçóquio ${ }^{1}$, M. Cristina Abduch ${ }^{1}$, Jeane Tsutsui $^{1}$, Wilson \\ Mathias $^{1}$, and José Carlos Nicolau ${ }^{1}$ \\ ${ }^{1}$ Universidade de Sao Paulo Instituto do Coracao
}

January 5, 2022

\begin{abstract}
BACKGROUND: Physical exercise adds benefits improving evolution of the ischemic heart disease, enhancing individual functional capacity and preventing ventricular remodeling. In this study we investigated the impact of a program of physical training started after an uncomplicated post-acute myocardial infarction (AMI) on the mechanics of left ventricle (LV) contraction. METHODS: A total of 53 patients were included, 27 of whom were randomized to a supervised training program (TRAINING group), and 26 to a CONTROL group, who received usual orientations for physical exercise after AMI. All patients underwent cardiopulmonary stress testing and an echocardiogram with speckle tracking technique to measure several parameters of LV contraction mechanics at 1 month and 5 months after AMI. RESULTS: No significant difference in the analysis of LV longitudinal, radial and circumferential strain parameters between groups after the training period was found. After the training program, analysis of torsional mechanics demonstrated a reduction in the LV basal rotation of the TRAINING group in comparison to the CONTROL group (TRAINING, $-5.9 \pm 2.3$ vs CONTROL, $-7.5 \pm 2.9^{\circ} ; P=0.03$ ), and in the basal rotational velocity ( TRAINING, $-53.6+-18.4$ vs CONTROL, $-68.8+-22.1 \mathrm{o} / \mathrm{s} ; P=0.01$ ), twist velocity (TRAINING, $127.4+-32.2$ vs CONTROL, $149.9+-35.9$ vs o/s; $P=0.02$ ) and torsion (TRAINING, $2.4+-0.4$ vs CONTROL, $2.8+-0.8$ vs o/cm; $P=0.02$ ). CONCLUSIONS: Physical activity did not cause a significant improvement in LV longitudinal, radial and circumferential deformation parameters. However, the exercise had a significant impact on the LV torsional mechanics, composed particularly of a reduction in basal rotation, twist velocity, torsion and torsional velocity which can be interpreted as a ventricular "torsion reserve" in this population.
\end{abstract}

\section{Introduction}

The benefit of exercise post-acute myocardial infarction (AMI) has been investigated for several years and the vast majority of publications have demonstrated a better prognosis related to this practice. ${ }^{1-7}$. Physical activity post-AMI is associated with better clinical evolution, with increased functional capacity and less progression to heart failure, preventing malefic cardiac remodeling. ${ }^{4,}$ 8-10

This benefit has been mainly demonstrated in cases of AMI that result in greater contractile involvement of the LV causing moderate to severe systolic dysfunction (LVEF $<40 \%$ ). ${ }^{11-18}$ However, with greater dissemination of information regarding the recognition of AMI "alarm symptoms", greater promptness of care, evolution of the therapies employed, less impairment of cardiac contraction after AMI has become more frequent. So, observation of ischemic cardiomyopathy with preserved LV systolic function or mild dysfunction (LVEF $45 \%$ to $55 \%$ ) is very common nowadays in the general population. Regarding to all this, little is known about the real benefit of exercise on LV remodeling and objective effect on LV mechanics of contraction in this population.

Echocardiogram with speckle tracking technique can perform a comprehensive study of LV contraction mechanics, which is characterized by longitudinal apex-to-base shortening in association to segmental rota- 
tions and ventricular torsion. This analysis adds numerous data over a simple determination of LV ejection fraction. ${ }^{19-25}$

The present study aims to test the hypothesis that cardiovascular rehabilitation under a supervised exercise program, in an uncomplicated post-infarction population, would have a positive impact on the mechanics of LV contraction.

\section{Methods}

\section{Study Design and Population}

This was a prospective, longitudinal, randomized and controlled study. Patients with uncomplicated AMI admitted to the Acute Coronary Disease Unit of the Heart Institute of the Sao Paulo Medical School (InCor/HCFMUSP), who agreed to participate in the study, were randomized to 2 groups (TRAINING and CONTROL), in the proportion 1:1 according to the following protocol: Time 0 : still hospitalized, all patients, after due explanations, signed the informed consent for inclusion in the study. Time 1 : all participants returned 1 month after AMI and were submitted to a 2D-STE echocardiogram and cardiopulmonary exercising testing (CPX). After this, participants randomized to the TRAINING group were included in a weekly supervised physical training program, twice a week, for 4 months, at the Rehabilitation Lab, while individuals randomized to the CONTROL group received usual recommendations for physical activity at home. Time 2 : at the end of the fourth month ( $5^{\text {th }}$ month after the AMI event), all patients repeated 2D-STE echo and CPX. This design is provided in the Supplementary Data I.

Inclusion criteria were: age over 18; hospitalization for spontaneous AMI with or without ST-segment elevation, established according to the $3^{\text {rd }}$ Universal Definition of $\mathrm{AMI}^{26}$; clinically and hemodynamically stable; left ventricular ejection fraction (LVEF) [?] 0.45 and Killip classes I or II. The exclusion criteria were: any condition that contraindicated physical activity; regular physical activity practitioners prior to the event; LV systolic dysfunction (LVEF $<45 \%$ ); Killip classes III or IV; irregular heart rhythm; cardiac pacemaker; chronic kidney disease characterized by creatinine clearance $<30 \mathrm{ml} / \mathrm{min} / \mathrm{m}^{2}$; limited echocardiographic window for analysis.

The present protocol was in accordance with the recommendations contained in the Declaration of Helsinki and was approved by the institutional Scientific and Ethics committees. All the patients signed the informed consent form before inclusion in this protocol.

\section{Conventional Echocardiogram}

The echocardiogram was performed by a single operator blind to the allocation group. Commercially available echocardiographic equipment (Vivid E9; GE Medical Systems, Milwaukee, WI, USA) was used, equipped with linear broadband transducers with a frequency of 5-2 MHz. Cavity dimensions were measured from longitudinal parasternal view. The systolic and diastolic cavity volumes, and LVEF, were calculated using Simpson's modified biplanar method, as recommended by the American Society of Echocardiography. ${ }^{27}$ The LV segmental contraction analysis was performed through wall motion score index calculation (WMSI). Valve flow analysis and LV diastolic function were was performed according to current guidelines. ${ }^{28-30}$

\section{STE Image Acquisition and Analysis}

For acquisition of 2D images for STE analysis, the machine was adjusted to acquire 3 cardiac cycles, with frame rate between 40-80 frames/s. Images were obtained in the parasternal short axis (PSAX) in its 3 main levels: basal (mitral valve), mid (papillary muscles) and apical. LV apical window was composed by: longitudinal (APLAX), four (A4C) and two chambers (A2C) views.

Off-line analyses were performed using the software EchoPAC, version v20.1 (GE Medical Systems, Milwaukee, WI, USA). The parameters evaluated were: strain $(\varepsilon, \%)$ and strain rate $\left(\varepsilon^{\prime}, \mathrm{s}^{-1}\right)$; maximum basal and apical rotations $\left(\right.$ VErot,$\left.{ }^{\circ}\right)$ and their peak absolute rotational velocities (VErot-v,${ }^{\circ} / \mathrm{s}$ ), LV twist $\left(\mathrm{VEtw},{ }^{\circ}\right.$ ) and torsion (VEtor, ${ }^{\circ} / \mathrm{cm}$ ), and their velocities (VEtw-v, $\%$ s; VEtor-v, $\%$ s.cm). LV twist was calculated as the net absolute difference between maximum apical and basal systolic peak rotations (VEtw = apical VErot - 
basal VErot) and torsion as the twist normalized to LV longitudinal length. By convention, baseline rotation values were negative, and apical, positive (figure 1). ${ }^{21}$

\section{Cardiopulmonary Exercise Testing (CPX)}

The CPX was performed on an electromagnetic cycle ergometer (Medfit 400L, Medical Fitness Equipment, Maarn, Netherlands), following a ramp protocol, with a speed of 60 revolutions per minute (rpm) and workload increments from $10 \mathrm{w}$ to $20 \mathrm{w}$ per minute, until achieving physical exhaustion. Participants were connected to a valve and trachea system (Vmax Spectra Series, SensorMedics Corp, Yorba Linda, CA, USA) where pulmonary ventilation (PV), $\mathrm{O}_{2}$ and $\mathrm{CO}_{2}$ concentrations were measured with calculation of oxygen consumption $\left(\mathrm{VO}_{2}\right)$ and $\mathrm{CO}_{2}$ production. When the patient achieved exhaustion, the anaerobic threshold (AT) and respiratory compensation point (RCP) were determined, both used to prescribe the intensity of physical training.

\section{Statistical Analysis}

Continuous data are presented as means $\pm \mathrm{SD}$ or median and interquartile ranges, according to normality of distribution assessed by the Shapiro-Wilks test. Comparisons of continuous variables between groups were performed using the Student's t test (when Gaussian distribution) or Mann-Whitney test (when non-Gaussian distribution) for independent samples. For categorical variables, the Chi-square test $\left(\chi^{2}\right)$ or Fisher's exact test, when indicated, were used. The within-group comparison of means was performed using the paired Student's t test. Finally, comparison of the "delta" $(\Delta)$ of values obtained from the follow-up of each group was done by the unpaired Student's t test.

Bivariate linear regression was performed to assess correlation between main variables of the CPX and data obtained from the echocardiogram-STE.

All tests were 2 -tailed and a $P$ value $<0.05$ was considered statistically significant. Statistical analyses were done using the SPSS 25.0 software for Macintosh (SPSS Inc, Chicago, IL).

\section{Inter- and Intraobserver Reproducibility}

Analysis of data reproducibility was done from reanalyses of 10 patients to assess inter- and intraobserver variability with determination of intraclass correlation coefficient (ICC) and variation coefficient (CV).

As reported in other echo labs, the correlations were considered excellent for longitudinal parameters (strain and SR), twist and torsion, and, in general, satisfactory for the parameters of transverse strain analyses (circumferential and radial). Results are provided in the Supplementary Data II.

\section{Results}

\section{Clinical Characteristics}

From November 2016 to March 2019, a total of 76 patients were enrolled. Of these, 23 were excluded for several reasons, with poor adherence to train the main reason (figure 2). The final study population consisted of 53 participants, 27 of whom were radomized and included to the TRAINING group and 26 to the CONTROL group.

The clinical characteristics of the population are shown in table 1 . The mean age in the TRAINING and CONTROL groups were $60 \pm 9$ years (20 males; $74 \%$ ) and $59 \pm 11$ years (19 males; $73 \%$ ), respectively.

There were no significant differences between groups regarding the variables related to CAD, in-hospital treatment, risk and severity of the ischemic event.

The average adherence of patients in the TRAINING group to the exercise program was $28.0 \pm 6.8$ sessions (minimum of 15 and maximum of 39 sessions), reaching an overall adherence of $88 \%$.

\section{Cardiopulmonary Exercise Testing (CPX)}


Table 2 provides the CPX main data. After the training period, the TRAINING group showed a significant increase in exercise duration (baseline $524 \pm 95$ vs $4 \mathrm{M} 636 \pm 131$ secs; $P=0.001$ ), maximum workload achieved (baseline $126 \pm 49$ vs $4 \mathrm{M} 151 \pm 65 \mathrm{~W} ; P=0.007$ ) and peak $\mathrm{VO}_{2}$ (baseline $21.7 \pm 5.2$ vs $4 \mathrm{M} 23.4 \pm 5.9$ $\mathrm{ml} / \mathrm{kg} / \mathrm{min} ; P=0.04)$. In the CONTROL group a significant increase in exercise duration was found (baseline, $491 \pm 93$ vs $4 \mathrm{M}, 593 \pm 93$ secs; $P=0.006)$.

At the end of the 4-month follow-up, no differences were observed in the variations (deltas) of the values over the several parameters measured between the groups. Analyses of exercise duration and $\mathrm{VO}_{2}$ deltas are provided in the Supplementary Data III.

\section{Conventional Echocardiogram}

Data from conventional echocardiogram are shown in table 2. A slight absolute difference in LA dimension at 4 months in the CONTROL group was noted (baseline, $37.3+-3.7$ vs $4 \mathrm{M}, 36.6+-3.4 \mathrm{~mm} ; P=0.02$ ). No other significant differences were found, including comparisons of deltas of all of these parameters between groups.

\section{Analysis of LV Contraction Mechanics}

\section{Apical View}

\section{Strain and Strain Rate (SR)}

Data is provided in table 3. No significant differences in the intergroup deltas of these parameters in response to exercise was found at the end of the follow-up. GLS and GLSR graphs are provided in the Supplementary Data IV.

\section{Transversal Axis}

\section{Circumferential and Radial Strain and SR, and Rotations}

Data obtained from the LV transversal axis analysis is presented in table 4. No significant difference in the deltas of circumferential and radial strain or SR values was found after the follow-up between groups. Referring to rotational mechanics, at the end of the training period, the TRAINING group had lower values of rotation of the basal segments compared to the CONTROL group (TRAINING 4M, -5.9+-2.3 vs CONTROL $4 \mathrm{M},-7.5+-2.9^{\circ} ; P=0.03$ ). There was a borderline significant difference of the delta of this parameter between groups (TRAINING, $0.8+-3.1$ vs CONTROL, $-0.8+-2.7^{\circ} ; P=0.05$ ). Additionally, lower basal rotational velocity values were also observed in the TRAINING group (TRAINING 4M, -53.6+-18.4 vs CONTROL $4 \mathrm{M},-68.8+-22.1 \mathrm{o} / \mathrm{s} ; P=0.01$ ), also with a trend of the delta toward a statistically significant difference (TRAINING $4.2+-21.5$ vs CONTROL $-8.1+-24.0^{\circ} / \mathrm{s} ; P=0.06$ ), as presented in figure 3 and Supplementary Data V.

\section{Twist and Torsion}

The results of the LV twist and torsion analyses are shown in table 4. At the end of the 4-month follow-up, compared to the CONTROL group, the TRAINING group showed significantly lower values of twist velocity (TRAINING 4M, 127.4+-32.2 vs CONTROL 4M, 149.9+-35.9; $P=0.02$ ) and torsion (TRAINING 4M, $2.4+-0.4 \mathrm{o} / \mathrm{cm}$ vs CONTROL $4 \mathrm{M}, 2.8+-0.8 ; P=0.02$ ). However, none of the deltas of all torsional mechanics parameters achieved a statistically significant difference (Supplementary Data VI).

\section{Correlation analysis}

Bivariate linear regression were performed to assess correlation between the $\mathrm{VO}_{2}$ deltas (4 months - baseline) obtained from CPX, and the deltas of several echocardiographic parameters. There were only poor and nonsignificant correlations. Results are provided in the Supplementary Data VII.

\section{Discussion}


This present study investigated the hypothesis that cardiovascular rehabilitation with a supervised exercise program would have a positively impact on the LV contraction mechanics in a population after an uncomplicated AMI. To date, to the best of our knowledge, there is no other similar study that investigated this hypothesis in such a detailed way, considering the large number of LV contraction parameters which comprise ventricular systolic mechanics performed in our study.

Regarding the analysis of longitudinal, circumferential and radial deformation, in a general analysis, the TRAINING group was not superior to the CONTROL group up to 4 months post-AMI.

However, we identified a very interesting finding in relation to the LV torsional mechanics. Compared to the CONTROL group, the TRAINING group showed significantly lower values of rotation and rotational velocity of the LV basal segments, as well as lower values of twist velocity, torsion and torsional velocity after the 16-week training period. Similar results were also observed by McGregor et al. in their elegant exploratory study. ${ }^{31}$ These authors also described a reduction in LV twist and twist velocity after 10-weeks of physical training sessions, twice a week, in a similar population who suffered an AMI and still maintained a preserved LV function (LVEF > 50\%). Exploring their data, this final result on LV twist was linked to a reduction in both basal and apical rotations. Finally, similar to our study, they did not found a significant positive impact on LV strain (longitudinal, circumferential or radial) determined by exercise.

Extrapolating to highly-trained athletes, despite some contrasts in the findings, studies point to a common and final real impact of exercise on LV torsional mechanics. Stohr et al. described a reduction of apical rotation and LV twist in individuals with high aerobic fitness. ${ }^{32}$ The same was found by Nottin et al. ${ }^{33}$ studying elite cyclist and Zocalo et al. ${ }^{34}$ assessing professional soccer players. A reduction of LV rates of basal and apical rotations, and torsional rate, were described. Weiner et al. showed also interesting findings with competitive rowing athletes. They described a so called "phasic phenomenon" in a program of high level physical activity, comprised by an acute phase of augmentation of LV twist, followed by a subsequent and chronic reduction of this parameter. ${ }^{35}$ Driven by this knowledge, it is valid to postulate that a possible greater enhancement of LV twist as a result during exercise could represent a major efficiency of systole in these individuals. ${ }^{36,37}$

The design of the myocardial muscle architecture most accepted by the scientific community was proposed by Dr. Francisco Torrent-Guasp who described the heart as a muscle band "folded" in double helix, with oblique endocardial fibers right-handed toward LV base and the apical epicardial fibers, more robust, with an oblique

and left-handed helix. ${ }^{38,39}$. Physiologically, as a result of this myocardial tissue conformation, after a brief counterclockwise rotation of the isovolumetric contraction phase, the base of LV contracts predominantly in clockwise rotation, opposed to the counterclockwise rotation apical, also after a slight initial clockwise rotation. This resulting opposition of rotations is the wringing motion of LV (twist or torsion). In terms of energy expenditure, this architecture would be a more efficient form of contraction, as well as having a more homogeneous distribution of cavity wall stress, with less myocardial oxygen consumption, compared to a simple radial LV cavity contraction. ${ }^{36}$

As in our study, McGregor et al. did not find a positive impact of exercise on longitudinal strain. Despite the good accuracy and more robust data in the literature on longitudinal strain, LV twist proved to be a more sensitive parameter for evaluating LV global systolic gain in response to physical exercise in this population.

\section{Limitations}

First, the subjectivity inherent to the echocardiogram exam can lead to quantification bias. Small variations in the acquisition at LV apical level, which do not have an anatomical marker, can result in distorted values. Image was acquired as the maximum apical SAX view achieved with still a clear visibility of the myocardial segments. Additionally, we created another criterion to ratify the correct acquisition, which was the visualization of at least a trend of a counterclockwise rotation of these segments, which would be physiologically expected. Finally, it is important to remind that the examiner was blinded to the patient's group allocation. 
Second, in this study we used the 2D-STE technique, which would have the limitation of detecting offplane movement of the speckles, causing eventual values underestimation. Theoretically, the use of the three-dimensional methodology (3D-STE) would have the possibility to correct this limitation.

Finally, the relatively small sample size, short duration of the exercise program and the number of training sessions for patients allocated to the TRAINING group (twice a week), with the aggravation of low adherence of some patients to the program, may have decreased the power of the study to demonstrate possible intergroup differences.

\section{Conclusions}

In this study, we investigated the hypothesis that cardiovascular rehabilitation after an uncomplicated AMI, through a supervised program of 16 weeks of physical exercise, would have a positive impact on the LV contraction mechanics. Despite the known benefits of exercise in the overall health of practicing individuals, there was no significant improvement in the LV longitudinal, radial and circumferential deformation parameters. However, exercise had a significant impact on the LV torsional mechanics, particularly composed of reduced basal rotation, twist velocity, torsion and torsional velocity. This outcome can be interpreted as a "LV torsional reserve" in individuals who exercised in post-AMI, possibly a physiologically more efficient torsional mechanics emerging from routine physical exercise.

\section{Abbreviations}

CAD, coronary artery disease; AMI, acute myocardial infarction; LVEF, left ventricle ejection fraction; STE, speckle tracking echocardiography; ANS, autonomic nervous system; CPX, cardiopulmonary exercising testing; PSAX, parasternal short axis; APLAX, apical longitudinal view/3 chamber; A4C, apical fourchamber view; A2C, apical two-chambers view; GLS, global longitudinal strain; GLSR, global longitudinal strain rate; GCS, global circumferential strain; GCSR, global circumferential strain rate; GRS, global radial strain; GRSR, global radial strain rate.

\section{Conflicts of interests}

None.

\section{Funding sources}

This research did not receive any specific grant from funding agencies in the public, commercial, or not-forprofit sectors.

\section{Data Availability}

The Authors confirm that the data supporting the findings of this study are available within the article and its Supplementary material. Raw data tha support the findings of this study are available from the corresponding author upon reasonable request.

\section{REFERENCES}

1. Suaya JA, Stason WB, Ades PA, et al. Cardiac rehabilitation and survival in older coronary patients. $J$ Am Coll Cardiol . 2009;54:25-33.

2. Oldridge NB, Guyatt GH, Fischer ME, et al. Cardiac rehabilitation after myocardial infarction. Combined experience of randomized clinical trials. JAMA . 1988;260:945-50.

3. O'Connor GT, Buring JE, Yusuf S, et al. An overview of randomized trials of rehabilitation with exercise after myocardial infarction. Circulation . 1989;80:234-44.

4. Taylor RS, Brown A, Ebrahim S, et al. Exercise-based rehabilitation for patients with coronary heart disease: systematic review and meta-analysis of randomized controlled trials. Am J Med . 2004;116:682-92.

5. Clark AM, Hartling L, Vandermeer B, et al. Meta-analysis: secondary prevention programs for patients with coronary artery disease. Ann Intern Med . 2005;143:659-72. 
6. Billman GE, Schwartz PJ, Stone HL. The effects of daily exercise on susceptibility to sudden cardiac death. Circulation . 1984;69:1182-9.

7. Hull SS Jr., Vanoli E, Adamson PB, et al. Exercise training confers anticipatory protection from sudden death during acute myocardial ischemia. Circulation . 1994;89:548-52.

8. McGregor G, Gaze D, Oxborough D, et al. Reverse left ventricular remodeling: effect of cardiac rehabilitation exercise training in myocardial infarction patients with preserved ejection fraction. Eur J Phys Rehabil Med . 2016;52:370-8.

9. Hammill BG, Curtis LH, Schulman KA, et al. Relationship between cardiac rehabilitation and long-term risks of death and myocardial infarction among elderly Medicare beneficiaries. Circulation . 2010;121:63-70.

10. Leon AS, Franklin BA, Costa F, et al. Cardiac rehabilitation and secondary prevention of coronary heart disease: an American Heart Association scientific statement from the Council on Clinical Cardiology (Subcommittee on Exercise, Cardiac Rehabilitation, and Prevention) and the Council on Nutrition, Physical Activity, and Metabolism (Subcommittee on Physical Activity), in collaboration with the American association of Cardiovascular and Pulmonary Rehabilitation.Circulation . 2005;111:369-76.

11. Giannuzzi P, Temporelli PL, Corra U, et al. Antiremodeling effect of long-term exercise training in patients with stable chronic heart failure: results of the Exercise in Left Ventricular Dysfunction and Chronic Heart Failure (ELVD-CHF) Trial. Circulation . 2003;108:554-9.

12. Belardinelli R, Georgiou D, Cianci G, et al. Effects of exercise training on left ventricular filling at rest and during exercise in patients with ischemic cardiomyopathy and severe left ventricular systolic dysfunction. Am Heart J . 1996;132:61-70.

13. Otsuka Y, Takaki H, Okano Y, et al. Exercise training without ventricular remodeling in patients with moderate to severe left ventricular dysfunction early after acute myocardial infarction. Int $J$ Cardiol . $2003 ; 87: 237-44$.

14. Sullivan MJ, Higginbotham MB, Cobb FR. Exercise training in patients with severe left ventricular dysfunction. Hemodynamic and metabolic effects. Circulation . 1988;78:506-15.

15. Zhang YM, Lu Y, Tang Y, et al. The effects of different initiation time of exercise training on left ventricular remodeling and cardiopulmonary rehabilitation in patients with left ventricular dysfunction after myocardial infarction. Disabil Rehabil . 2016;38:268-76.

16. Haykowsky M, Scott J, Esch B, et al. A meta-analysis of the effects of exercise training on left ventricular remodeling following myocardial infarction: start early and go longer for greatest exercise benefits on remodeling. Trials . 2011;12:92.

17. Giannuzzi P, Temporelli PL, Corra U, et al. Attenuation of unfavorable remodeling by exercise training in postinfarction patients with left ventricular dysfunction: results of the Exercise in Left Ventricular Dysfunction (ELVD) trial. Circulation . 1997;96:1790-7.

18. Koizumi T, Miyazaki A, Komiyama N, et al. Improvement of left ventricular dysfunction during exercise by walking in patients with successful percutaneous coronary intervention for acute myocardial infarction. Circ J . 2003;67:233-7.

19. Lima MSM, Villarraga HR, Abduch MC, et al. Comprehensive left ventricular mechanics analysis by speckle tracking echocardiography in Chagas disease. Cardiovasc Ultrasound . 2016;14:20.

20. Abduch MC, Alencar AM, Mathias W Jr., et al. Cardiac mechanics evaluated by speckle tracking echocardiography. Arq Bras Cardiol . 2014;102:403-12.

21. Mor-Avi V, Lang RM, Badano LP, et al. Current and evolving echocardiographic techniques for the quantitative evaluation of cardiac mechanics: ASE/EAE consensus statement on methodology and indications endorsed by the Japanese Society of Echocardiography. Eur J Echocardiogr . 2011;12:167-205. 
22. Abduch MC, Salgo I, Tsang W, et al. Myocardial deformation by speckle tracking in severe dilated cardiomyopathy. Arq Bras Cardiol . 2012;99:834-43.

23. Edvardsen T, Helle-Valle T, Smiseth OA. Systolic dysfunction in heart failure with normal ejection fraction: Speckle-tracking echocardiography. Progress in Cardiovascular Diseases . 2006;49:207-214.

24. Govind SC, Gadiyaram VK, Quintana M, et al. Study of left ventricular rotation and torsion in the acute phase of ST-elevation myocardial infarction by speckle tracking echocardiography.Echocardiography . 2010;27:45-9.

25. Feigenbaum H, Mastouri R, Sawada S. A practical approach to using strain echocardiography to evaluate the left ventricle. Circ J . 2012;76:1550-5.

26. Thygesen K, Alpert JS, Jaffe AS, et al. Third universal definition of myocardial infarction. Circulation . 2012;126:2020-35.

27. Lang RM, Badano LP, Mor-Avi V, et al. Recommendations for cardiac chamber quantification by echocardiography in adults: an update from the American Society of Echocardiography and the European Association of Cardiovascular Imaging. J Am Soc Echocardiogr . 2015;28:1-39.e14.

28. Mitchell C, Rahko PS, Blauwet LA, et al. Guidelines for Performing a Comprehensive Transthoracic Echocardiographic Examination in Adults: Recommendations from the American Society of Echocardiography. J Am Soc Echocardiogr . 2019;32:1-64.

29. Nishimura RA, Otto CM, Bonow RO, et al. 2017 AHA/ACC Focused Update of the 2014 AHA/ACC Guideline for the Management of Patients With Valvular Heart Disease: A Report of the American College of Cardiology/American Heart Association Task Force on Clinical Practice Guidelines. Circulation . 2017;135:e1159-e1195.

30. Nagueh SF, Smiseth OA, Appleton CP, et al. Recommendations for the Evaluation of Left Ventricular Diastolic Function by Echocardiography: An Update from the American Society of Echocardiography and the European Association of Cardiovascular Imaging. J Am Soc Echocardiogr . 2016;29:277-314.

31. McGregor G, Stohr EJ, Oxborough D, et al. Effect of exercise training on left ventricular mechanics after acute myocardial infarction-an exploratory study. Ann Phys Rehabil Med . 2018;61:119-124.

32. Stohr EJ, McDonnell B, Thompson J, et al. Left ventricular mechanics in humans with high aerobic fitness: adaptation independent of structural remodelling, arterial haemodynamics and heart rate. $J$ Physiol . 2012;590:2107-19.

33. Nottin S, Doucende G, Schuster-Beck I, et al. Alteration in left ventricular normal and shear strains evaluated by 2D-strain echocardiography in the athlete's heart. J Physiol . 2008;586:4721-33.

34. Zocalo Y, Bia D, Armentano RL, et al. Assessment of training-dependent changes in the left ventricle torsion dynamics of professional soccer players using speckle-tracking echocardiography.Annu Int Conf IEEE Eng Med Biol Soc . 2007;2007:2709-12.

35. Weiner RB, DeLuca JR, Wang F, et al. Exercise-Induced Left Ventricular Remodeling Among Competitive Athletes: A Phasic Phenomenon. Circ Cardiovasc Imaging . 2015;8.

36. Beladan CC, Călin A, Roşca M, et al. Left ventricular twist dynamics: principles and applications. Heart . 2014;100:731-40.

37. Sengupta PP, Khandheria BK, Narula J. Twist and untwist mechanics of the left ventricle. Heart Fail Clin . 2008;4:315-24.

38. Torrent-Guasp F, Kocica MJ, Corno AF, et al. Towards new understanding of the heart structure and function. European Journal of Cardio-Thoracic Surgery . 2005;27:191-201. 
39. Buckberg GD, Weisfeldt ML, Ballester M, et al. Left ventricular form and function - Scientific priorities and strategic planning for development of new views of disease. Circulation . 2004;110:E333-E336.

\section{FIGURES LEGENDS}

Cover Image. A, example of apical rotation measurement. B, graphical representation of LV torsion. $\mathrm{LV}$, left ventricle; RV, right ventricle; AVC, aortic valve closure; D, LV length. C, LV basal rotation and rotational velocity.

Figure 1. A, example of apical rotation measurement. B, graphical representation of LV torsion. LV, left ventricle; RV, right ventricle; AVC, aortic valve closure; D, LV length.

Figure 2. Patient inclusion flowchart.

Figure 3. LV basal rotation and rotational velocity.

\section{TABLES}

Table 1. Baseline characteristics of the population

\begin{tabular}{|c|c|c|}
\hline Variables & Control $(n=26)$ & Training $(n=27)$ \\
\hline \multicolumn{3}{|l|}{ Demographics } \\
\hline Age, $y$ & $59 \pm 11$ & $60 \pm 9$ \\
\hline Gender M / F n (\%) & $19(73 \%) / 7(27 \%)$ & $20(74 \%) / 7(26 \%)$ \\
\hline $\mathrm{BMI}, \mathrm{kg} / \mathrm{m}^{2}$ & $27.4 \pm 3.9$ & $27.2 \pm 3.9$ \\
\hline Hypertension, n (\%) & $17(65 \%)$ & $13(48 \%)$ \\
\hline Diabetes Mellitus, n (\%) & $10(38 \%)$ & $6(22 \%)$ \\
\hline Smoker, n (\%) & $5(19 \%)$ & $10(37 \%)$ \\
\hline Sedentarism, n (\%) & $26(100 \%)$ & $27(100 \%)$ \\
\hline Previous AMI, n (\%) & $4(15 \%)$ & $3(11 \%)$ \\
\hline \multicolumn{3}{|l|}{ Treatment } \\
\hline Aspirin, n (\%) & $26(100 \%)$ & $27(100 \%)$ \\
\hline Clopidogrel, n (\%) & $24(92 \%)$ & $27(100 \%)$ \\
\hline Ticagrelor, n (\%) & $2(8 \%)$ & $0(0 \%)$ \\
\hline$\beta-\beta \lambda o \varsigma x \varepsilon \rho, \nu(\%)$ & $17(65 \%)$ & $23(85 \%)$ \\
\hline \multicolumn{3}{|l|}{ Event } \\
\hline STEMI, n (\%) & $14(54 \%)$ & $16(59 \%)$ \\
\hline NSTEMI, n (\%) & $12(46 \%)$ & $11(41 \%)$ \\
\hline $\begin{array}{l}\text { Troponin, ng/mL (median, } \\
\text { IQR) }\end{array}$ & $22(3-50)$ & $32(11-50)$ \\
\hline \multicolumn{3}{|l|}{ Risk Scores } \\
\hline TIMI STEMI score & $3.3 \pm 1.8$ & $2.7 \pm 1.8$ \\
\hline TIMI NSTEMI score & $3.7 \pm 1.3$ & $3.2 \pm 1.1$ \\
\hline GRACE (median, IQR) & $129(120-159)$ & $139(121-151)$ \\
\hline \multicolumn{3}{|l|}{ Risk Scores } \\
\hline TIMI STEMI score & $3.3 \pm 1.8$ & $2.7 \pm 1.8$ \\
\hline TIMI NSTEMI score & $3.7 \pm 1.3$ & $3.2 \pm 1.1$ \\
\hline GRACE (median, IQR) & $129(120-159)$ & $139(121-151)$ \\
\hline \multicolumn{3}{|l|}{ Treatment } \\
\hline Fibrinolysis, n (\%) & $4(15 \%)$ & $6(22 \%)$ \\
\hline Primary PTCA, n (\%) & $8(31 \%)$ & $8(30 \%)$ \\
\hline $\begin{array}{l}\text { Pharmaco-invasive strategy, } \\
\text { n (\%) }\end{array}$ & $3(12 \%)$ & $2(7 \%)$ \\
\hline BMS, n (\%) & $19(73 \%)$ & $23(85 \%)$ \\
\hline
\end{tabular}




\begin{tabular}{lll}
\hline Variables & Control $(\mathbf{n}=\mathbf{2 6})$ & Training $(\mathbf{n}=\mathbf{2 7})$ \\
\hline DES, $\mathbf{n}(\boldsymbol{\%})$ & $5(19 \%)$ & $1(4 \%)$ \\
\hline
\end{tabular}

Data expressed as mean $\pm \mathrm{SD}$, median (IQR, interquartile range; CI 95\%) or number (\%). BC indicates body surface; BMI, body mass index; CAD, coronary artery disease; CABG, coronary artery bypass grafting; ACEI, angiotensin I converting enzyme inhibitor; BRA, angiotensin II receptor blocker. STEMI indicates ST-segment elevation myocardial infarction; NSTEMI, Non-ST segment elevation myocardial infarction; CK$\mathrm{MB}, \mathrm{MB}$ fraction of the creatine kinase enzyme; PTCA, percutaneous transluminal coronary angioplasty; BMS, bare-metal stent; DES, drug-eluting stent. $P=\mathrm{NS}$ for all comparisons.

Table 2. Standard echocardiographic and cardiopulmonary stress testing variables.

\begin{tabular}{|c|c|c|}
\hline & CONTROL $_{-}$ & CONTROL \\
\hline Variáveis & Basal & $4 \mathrm{M}$ \\
\hline \multicolumn{3}{|l|}{ Echocardiogram } \\
\hline $\mathbf{L A}, \mathbf{m m}$ & $37.3 \pm 3.7$ & $36.6 \pm 3.4$ \\
\hline LVEDD, mm & $50.2 \pm 4.3$ & $50.0 \pm 4.3$ \\
\hline LVESD, mm & $33.1 \pm 5.0$ & $31.9 \pm 3.9$ \\
\hline LVEDV, ml & $99.9 \pm 23.2$ & $99.5 \pm 23.5$ \\
\hline LVESV, ml & $39.6 \pm 11.1$ & $42.0 \pm 10.2$ \\
\hline LVMI. $\mathrm{g} / \mathrm{m}^{2}$ & $90.7 \pm 18.6$ & $89,7 \pm 18.0$ \\
\hline LVEF, \% & $61.3 \pm 5.7$ & $60,0 \pm 5.1$ \\
\hline WMSI & $1.19 \pm 0.19$ & $1,16 \pm 0.19$ \\
\hline E Vel, cm/s & $81 \pm 24$ & $81 \pm 22$ \\
\hline e' Vel cm/s & $7.1 \pm 1.6$ & $7.2 \pm 1.8$ \\
\hline E/e' ratio & $12.1 \pm 5.3$ & $11.8 \pm 4.6$ \\
\hline \multicolumn{3}{|l|}{$C P X$} \\
\hline Exercise duration, secs & $491 \pm 93$ & $593 \pm 93$ \\
\hline Basal HR, bpm & $73 \pm 14$ & $70 \pm 11$ \\
\hline Max HR, bpm & $133 \pm 22$ & $134 \pm 15$ \\
\hline Basal SBP, mmHg & $124 \pm 11$ & $119 \pm 14$ \\
\hline Peak SBP, mmHg & $169 \pm 18$ & $176 \pm 16$ \\
\hline Maximal exercise workload, W & $109 \pm 43$ & $119 \pm 48$ \\
\hline Peak $\mathrm{VO}_{2}, \mathrm{ml} / \mathrm{kg} / \mathrm{min}$ & $20.6 \pm 4.6$ & $21.7 \pm 4.9$ \\
\hline $\mathrm{AT}, \mathrm{ml} / \mathrm{kg} / \mathrm{min}$ & $12.3 \pm 2.3$ & $13.7 \pm 2.4$ \\
\hline $\mathrm{VE}, \mathrm{L} / \mathrm{min}$ & $59.5 \pm 21.3$ & $59.4 \pm 19.5$ \\
\hline
\end{tabular}

Data expressed as mean \pm SD. LA, left atrium; LVEDD, LV end diastolic diameter; LVFSF, LV end systolic diameter; LVMI, LV mass index; LVEDV, LV end-diastolic volume; LVESV, LV end-sistolic volume; LVEF, LV ejection fraction; WMSI, wall motion score index; E Vel, transmitral E wave velocity; A Vel, transmitral A wave velocity; e' Vel, septal e' wave velocity; a' Vel, septal a' wave velocity. HR indicates heart rate; SBP, systolic blood pressure; $\mathrm{VO}_{2}$, oxygen consumption; AT, anaerobic threshold; VE, minute volume.

${ }^{*} P=0.02$ for comparison with CONTROL

$+P=0.04$ for comparison with CONTROL

Table 3. Result of LV strain and strain rate analysis. Analysis of the apical window (longitudinal views, 4 and 2 chambers). 


\begin{tabular}{|c|c|c|c|}
\hline & CONTROL & CONTROL & CONTROI \\
\hline $\begin{array}{l}\text { Variables } \\
A P L A X\end{array}$ & Basal & $4 \mathrm{M}$ & $\mathrm{P}$ \\
\hline LS, \% & $-17.5 \pm 3.3$ & $-18.6 \pm 2.5$ & 0.09 \\
\hline $\begin{array}{l}\mathbf{L S R}, \mathbf{1} / \mathbf{s} \\
A_{4} C\end{array}$ & $-0.98 \pm 0.16$ & $-1.04 \pm 0.20$ & 0.08 \\
\hline LS, \% & $-18.5 \pm 3.3$ & $-18.6 \pm 2.8$ & 0.91 \\
\hline 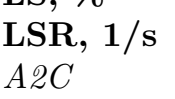 & $-1.01 \pm 0.23$ & $-1.00 \pm 0.20$ & 0.98 \\
\hline LS, \% & $-18.7 \pm 2.9$ & $-18.4 \pm 2.7$ & 0.36 \\
\hline $\begin{array}{l}\mathbf{L S R}, \mathbf{1} / \mathbf{s} \\
G L O B A L\end{array}$ & $-1.04 \pm 0.21$ & $-1.01 \pm 0.15$ & 0.24 \\
\hline $\mathrm{LS}, \%$ & $-18.3 \pm 2.7$ & $-18.5 \pm 2.5$ & 0.55 \\
\hline LSR, 1/s & $-1.00 \pm 0.18$ & $-1.02 \pm 0.17$ & 0.67 \\
\hline
\end{tabular}

Data expressed as mean \pm SD. APLAX, apical window, longitudinal view; A4C, apical window 4-chamber view; A2C, apical window 2-chamber view; LS, longitudinal strain; LSR, longitudinal strain rate.

Table 4. Analysis of ventricular contraction mechanics obtained in the short transverse LV axis (basal, mid and apical). LV twist and torsion mechanics.

\begin{tabular}{|c|c|c|c|}
\hline & CONTROL & CONTROL & CONTROL \\
\hline & Basal & $4 \mathrm{M}$ & $\mathrm{P}$ \\
\hline \multicolumn{4}{|l|}{$P S A X-$ Basal } \\
\hline CS, \% & $-17.0 \pm 3.9$ & $-18.0 \pm 3.1$ & 0.11 \\
\hline RS, \% & $39.7 \pm 21.0$ & $34.3 \pm 17.9$ & 0.13 \\
\hline $\mathrm{CSR}, 1 / \mathrm{s}$ & $-1.54 \pm 0.40$ & $-1.62 \pm 0.36$ & 0.42 \\
\hline RSR, 1/s & $2.32 \pm 0.92$ & $2.41 \pm 0.83$ & 0.68 \\
\hline Rot, degrees & $-6.7 \pm 3.2$ & $-7.5 \pm 2.9$ & 0.18 \\
\hline \multicolumn{3}{|l|}{$P S A X-M i d$} & 0.10 \\
\hline $\mathrm{CS}, \%$ & $-15.8 \pm 4.1$ & $-16.3 \pm 3.6$ & 0.55 \\
\hline RS, \% & $34.4 \pm 19.3$ & $39.4 \pm 18.0$ & 0.24 \\
\hline $\mathrm{CSR}, 1 / \mathrm{s}$ & $-1.44 \pm 0.25$ & $-1.43 \pm 0.26$ & 0.75 \\
\hline $\begin{array}{l}\mathbf{R S R}, \mathbf{1} / \mathbf{s} \\
P S A X-A \text { pex }\end{array}$ & $2.10 \pm 0.84$ & $2.31 \pm 1.11$ & 0.23 \\
\hline $\mathrm{CS}, \%$ & $-19.3 \pm 4.8$ & $-18.0 \pm 3.9$ & 0.16 \\
\hline $\mathrm{RS}, \%$ & $23.9 \pm 9.0$ & $19.1 \pm 8.2$ & 0.19 \\
\hline $\mathrm{CSR}, 1 / \mathrm{s}$ & $-1.53 \pm 0.40$ & $-1.48 \pm 0.42$ & 0.67 \\
\hline RSR, 1/s & $1.89 \pm 0.97$ & $1.80 \pm 0.91$ & 0.77 \\
\hline Rot, degrees & $15.5 \pm 6.5$ & $15.5 \pm 5.0$ & 0.95 \\
\hline \multicolumn{3}{|l|}{$G L O B A L$} & 0.43 \\
\hline $\mathrm{CS}, \%$ & $-17.4 \pm 3.5$ & $-17.4 \pm 2.6$ & 0.95 \\
\hline RS, \% & $30.8 \pm 12.7$ & $30.7 \pm 8.5$ & 0.94 \\
\hline CSR, 1/s & $-1.51 \pm 0.27$ & $-1.51 \pm 0.21$ & 0.97 \\
\hline RSR, 1/s & $2.10 \pm 0.58$ & $2.17 \pm 0.52$ & 0.57 \\
\hline \multicolumn{4}{|l|}{ TORSION } \\
\hline Twist ${ }^{\circ}$ & $22.2 \pm 7.1$ & $23.1 \pm 6.0$ & 0.46 \\
\hline Tw vel, $\% / \mathrm{s}$ & $145.5 \pm 32.3$ & $149.9 \pm 35.9$ & 0.53 \\
\hline
\end{tabular}




\begin{tabular}{llll}
\hline & CONTROL & CONTROL & CONTROL \\
\hline Torsion, $^{\circ} / \mathrm{cm}$ & $2.6 \pm 0.8$ & $2.8 \pm 0.8$ & 0.18 \\
$\mathrm{~T}^{\mathrm{N}} \mathrm{vel}, \mathrm{o} / \mathrm{s.cm}$ & $17.4 \pm 3.9$ & $18.5 \pm 5.2$ & 0.22 \\
\hline
\end{tabular}

Data expressed as mean \pm SD. PSAX-basal, parasternal short axis, basal; PSAX-mid, short axis, mid level; PSAX-apical, short axis, apical level; CS, circumferential strain; RS, radial strain; CSR, circumferential strain rate; RSR, radial strain rate; Rot, rotation; Rot Vel: rotational velocity. Tw vel, twist velocity; T vel, torsional vel.

${ }^{*} P=0.01$ for comparison with CONTROL

$+P=0.03$ for comparison with CONTROL

$++P=0.01$ for comparison with CONTROL

$\mathrm{Y}=P=0.02$ for comparison with CONTROL

$\mathrm{PS} P=0.02$ for comparison with CONTROL 


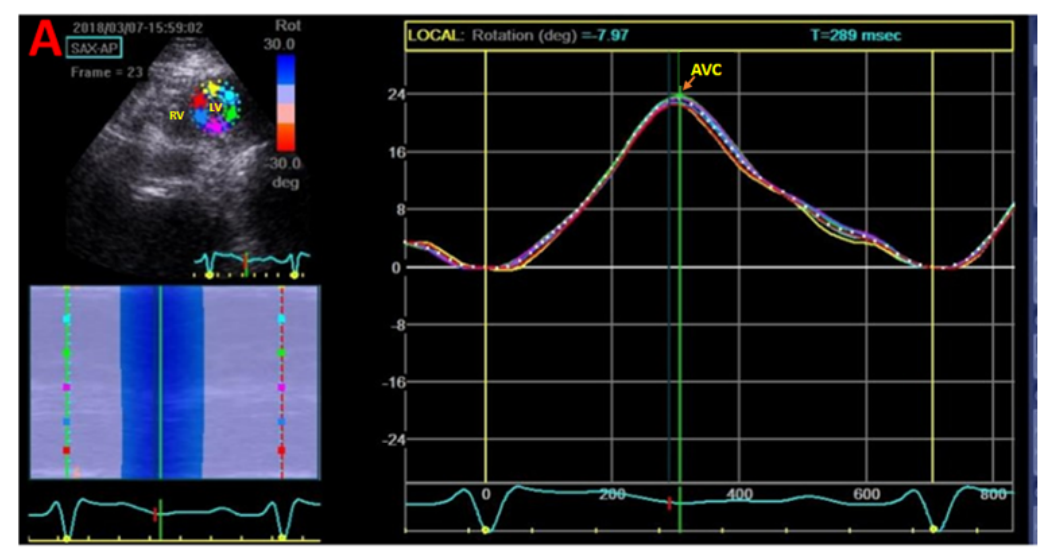

B

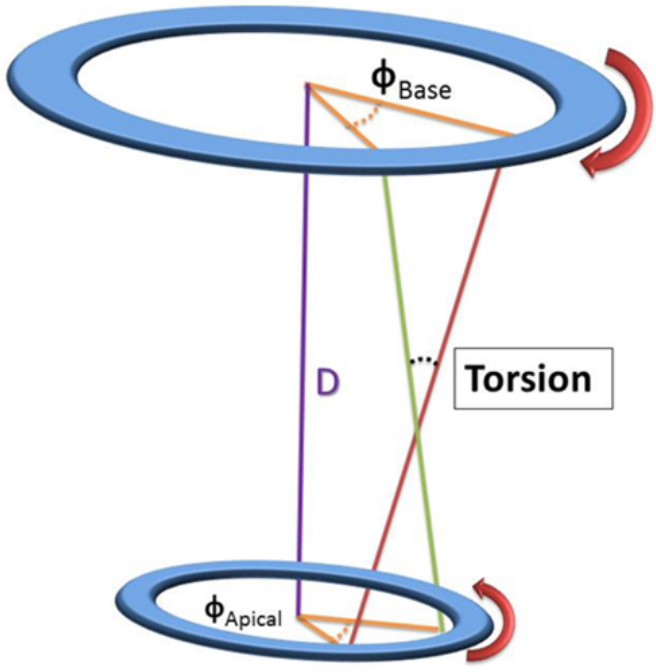

Figure 1. A, example of apical rotation measurement. B, graphical representation of $L V$ torsion. $L V$, left ventricle; $R V$, right ventricle; $\mathrm{AVC}$, aortic valve closure; D, LV length. 


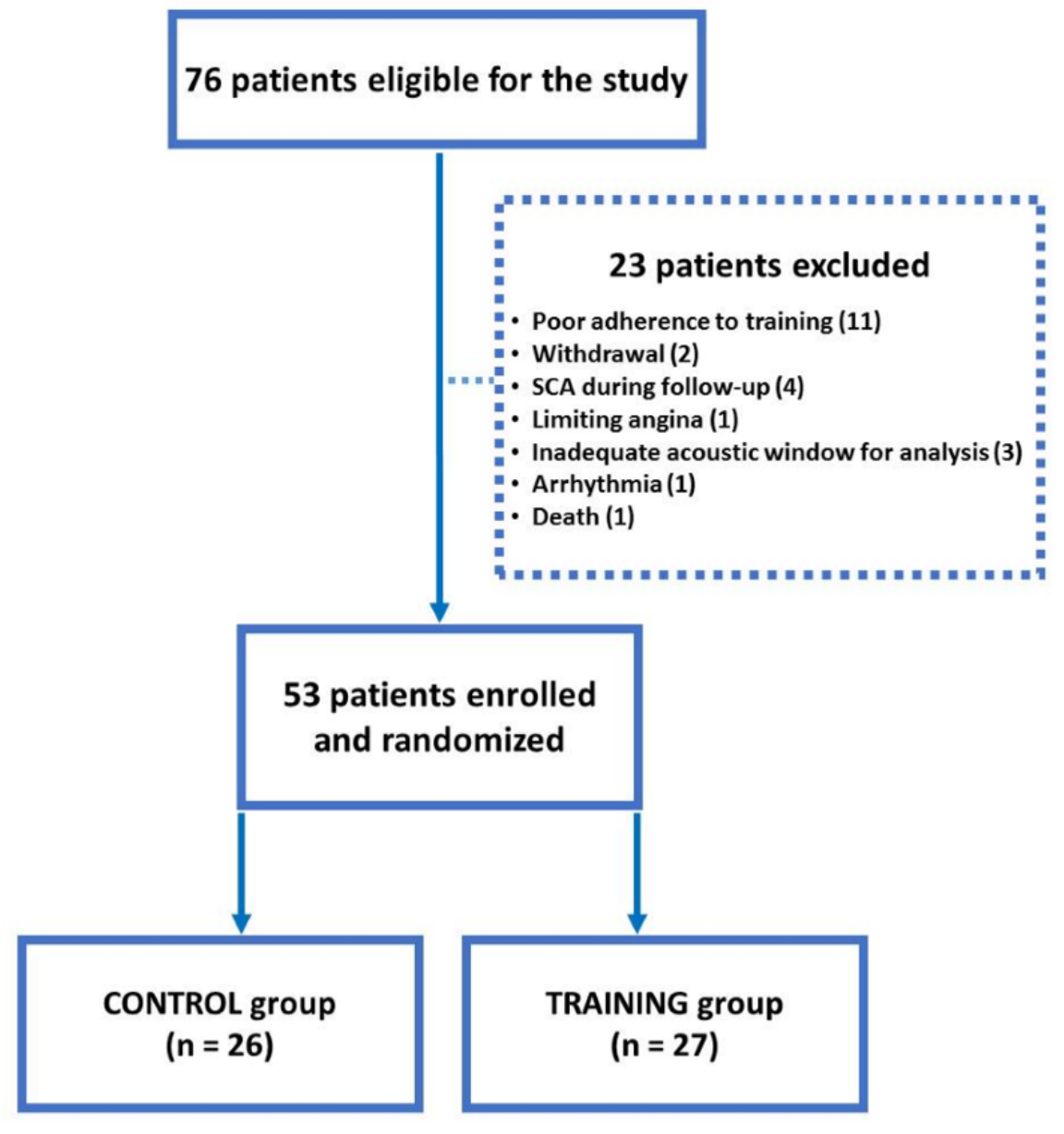

Figure 2. Patient inclusion flowchart. 

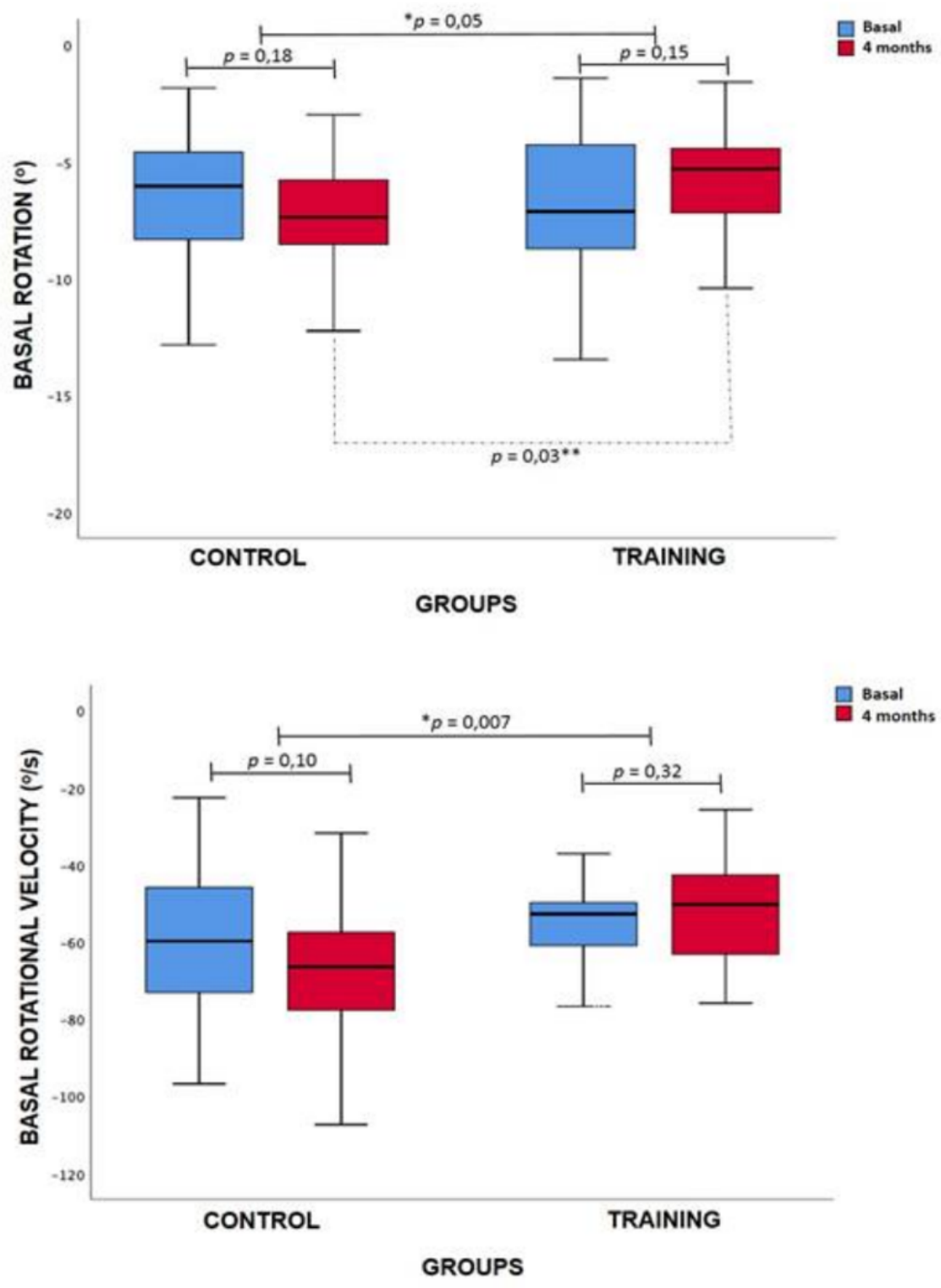

Figure 3. LV basal rotation and rotational velocity. 

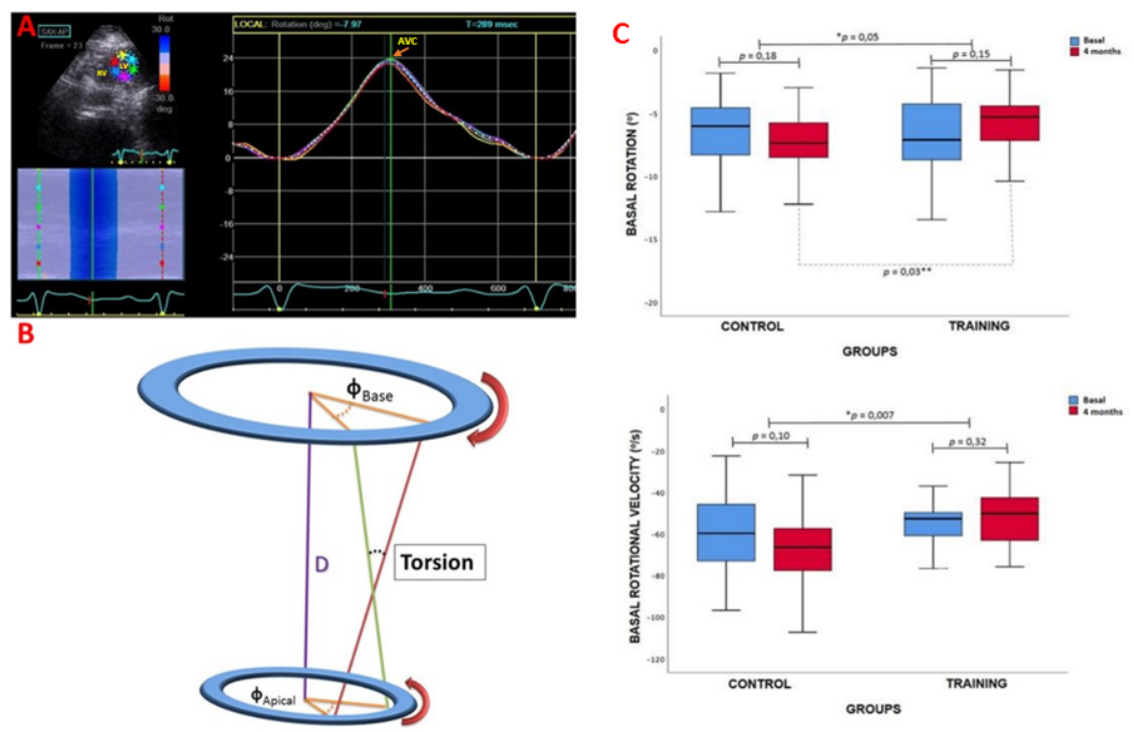

Cover Image. A, example of apical rotation measurement. B, graphical representation of LV torsion. LV, left ventricle; RV, right ventricle; AVC, aortic valve closure; D, LV length. C, LV basal rotation and rotational velocity. 